\title{
RAGE Architecture for Reusable Serious Gaming Technology Components
}

\author{
Wim van der Vegt, ${ }^{1}$ Wim Westera, ${ }^{1}$ Enkhbold Nyamsuren, ${ }^{1}$ \\ Atanas Georgiev, ${ }^{2}$ and Iván Martínez Ortiz ${ }^{3}$ \\ ${ }^{1}$ Open University of the Netherlands, Valkenburgerweg 177, 6419 AT Heerlen, Netherlands \\ ${ }^{2}$ Sofia University "St. Kliment Ohridski", Boulevard Tzar Osvoboditel 15, 1504 Sofia, Bulgaria \\ ${ }^{3}$ Complutense University of Madrid, Avenida de Séneca 2, 28040 Madrid, Spain
}

Correspondence should be addressed to Wim Westera; wim.westera@ou.nl

Received 8 December 2015; Revised 10 February 2016; Accepted 2 March 2016

Academic Editor: Michael Wimmer

Copyright (C) 2016 Wim van der Vegt et al. This is an open access article distributed under the Creative Commons Attribution License, which permits unrestricted use, distribution, and reproduction in any medium, provided the original work is properly cited.

For seizing the potential of serious games, the RAGE project-funded by the Horizon-2020 Programme of the European Commission-will make available an interoperable set of advanced technology components (software assets) that support game studios at serious game development. This paper describes the overall software architecture and design conditions that are needed for the easy integration and reuse of such software assets in existing game platforms. Based on the component-based software engineering paradigm the RAGE architecture takes into account the portability of assets to different operating systems, different programming languages, and different game engines. It avoids dependencies on external software frameworks and minimises code that may hinder integration with game engine code. Furthermore it relies on a limited set of standard software patterns and wellestablished coding practices. The RAGE architecture has been successfully validated by implementing and testing basic software assets in four major programming languages (C\#, C++, Java, and TypeScript/JavaScript, resp.). Demonstrator implementation of asset integration with an existing game engine was created and validated. The presented RAGE architecture paves the way for large scale development and application of cross-engine reusable software assets for enhancing the quality and diversity of serious gaming.

\section{Introduction}

The potential of nonleisure games (serious games) in industry, health, education, and the public administration sectors has been widely recognised. An increasing body of evidence is demonstrating the effectiveness of games for teaching and training [1]. While instructional scientists consider motivation as a main driver for effective learning [2-5], games are capable of amplifying the players' motivation by hooking and absorbing them in such a way that they can hardly stop playing [6]. This motivational power of games is ascribed to their dynamic, responsive, and visualised nature, which goes along with novelty, variation, and choice, effecting strong user involvement and providing penetrating learning experiences [6]. In addition, serious games allow for safe experimentation in realistic environments, stimulate problem ownership by role adoption, and allow for learning-by-doing approaches, which support the acquisition of tacit and contextualised knowledge [7]. Nevertheless, the complexity of serious game design may hamper the games' effectiveness [8]. In particular the subtle balance between game mechanics and pedagogical power is not self-evident [9]. Also, various authors $[1,10]$ note that many studies fail to evaluate the educational effectiveness of serious games in a rigorous manner and they call for more randomised controlled trials (involving comparisons between an experimental group and a control group) for increased scientific robustness. Still, the potential of games for learning is widely recognised, stimulating serious game development as a new branch of business.

For various reasons, however, seizing this potential has been problematic. The serious game industry displays many features of an emerging, immature branch of business: being 
scattered over a large number of small independent players, weak interconnectedness, limited knowledge exchange, absence of harmonising standards, a lot of studios creating their localised solutions leading to "reinventing the wheel," limited specialisations, limited division of labour, and insufficient evidence of the products' efficacies [11, 12]. Still, conditions for a wider uptake of serious games are favourable. PCs, game consoles, and handheld devices have become lowpriced commodities as are to a lesser extent advanced tools for game creation and the associated graphics design and media production.

In order to enhance the internal cohesion of the serious game industry sector the RAGE project (http://rageproject .eu/), which is Europe's principal research and innovation project on serious gaming in the Horizon-2020 Programme, makes available a diversity of software modules (software assets) for developing serious games easier, faster, and more cost-effectively. The pursued software assets cover a wide range of functionalities particularly tuned to the pedagogy of serious gaming, for example, in player data analytics, emotion recognition, stealth assessment, personalisation, game balancing, procedural animations, language analysis and generation, interactive storytelling, and social gamification. Game developers could then simply add the required assets to their project without the need to do all the programming themselves. For example, the stealth assessment asset would allow the game development team to easily incorporate diagnostic functionality that provides metrics for the progressive mastery of knowledge and skills, based on the tracking and processing of the players' behavioural patterns in the game.

Importantly, the creation of the game software assets is not an isolated technical endeavour, but instead it is positioned as a joint activity of multiple stakeholders represented in the RAGE consortium. In the consortium computer scientists and IT developers are working together with serious game developers, educational researchers, education providers, and end-users to make sure that the new technology components are practical and usable and create added pedagogical value. To this end the architecture presented in this paper is used for supporting the easy integration of assets in a set of serious games that will be developed in the project. The games and the assets included will be empirically tested for their effectiveness in large scale pilots with end-users. Hence, one of the major technical challenges of RAGE is to ensure interoperability of the software assets across the variety of game engines, game platforms, and programming languages that game studios have in use. The incorporation of the software assets should be as easy as possible (e.g., "plugand-play"), without enforcing specific standards or systems as to avoid principal adoption barriers. In addition, the software assets should allow for being grouped together into more complex aggregates, for example, combining an emotion recognition asset with a game-balancing asset. That is, the assets should comprise a coherent, component-based system that supports data interoperability between its elements.

This paper takes a technical perspective by describing and validating the RAGE software architecture that aims to accommodate the easy integration and reuse of such interoperable software assets in existing game platforms.
The RAGE architecture particularly addresses the structure and functioning of client-side assets. Since exactly client-side assets are supposed to be fully integrated in the game engine, noncompliance issues of assets are likely to occur clientside. This paper will explain the basic requirements and the proposed solution. It presents the proofs of concept that have been created for validating the RAGE architecture in four different programming languages, and it describes how the concrete technical issues encountered in these proofs were overcome.

\section{Related Work}

There are various existing efforts in promoting reusability in both serious games and leisure games. The Unity Asset Store (https://www.assetstore.unity3d.com/) is an example of a successful online marketplace for game objects. Most of the objects are media objects (e.g., terrains, audio, buildings, and weapons), but an increasing number of software modules (e.g., analytics, cloud backend, and game AI) are becoming available. Unfortunately, most of the software objects can only be reused in the Unity game engine. Various other online platforms offer reusable game objects, for instance, the Guru asset store (https://en.tgcstore.net/), Game Salads (http:// gshelper.com/), GameDev Market (https://www.gamedevmarket.net/), Unreal Marketplace (https://www.unrealengine.com/marketplace), and Construct 2 (https://www.scirra.com/store), but their main focus is on user-interface objects and templates.

At a more abstract level Folmer [13] proposed a reference architecture for games. The architecture consists of several layers, such as game interface layer and domain specific layer, and it identifies reusable components within each layer. A similar idea proposed by Furtado et al. [14] describes a software product line-based approach for creating reusable software modules specific to a particular (sub)domain of games. These modules include domain specific reference architectures and languages. The current paper, however, is little concerned with defining a reusability framework for the entire process of game development. Instead, it proposes a "reference architecture" for a particular niche within serious game development that covers enhanced pedagogical functionality. Consequently, while attempting to simplify the reuse of pedagogical components, the RAGE architecture interferes as little as possible with established game development processes.

A recent reusability framework targeting serious games $[15,16]$ relies on a service-oriented architecture (SOA). Within this framework, domain independent features commonly used in serious games are encapsulated into components and implemented as services. SOA offers several advantages such as decoupling from implementation details and a high degree of reusability. However, the SOA approach goes with several limitations, for instance, the requirement of being constantly online, diminished flexibility such as customisation and configuration of services by service consumers, reduced system performance due to additional overheads associated with SOA, and network calls. 
A more versatile reference architecture is needed to ensure seamless interoperability of the software assets across different game engines, game platforms, and programming languages, while the limitations of SOA should be avoided as much as possible.

\section{Starting Points}

3.1. The Asset Concept. The idea of making available a set of software assets seems to neglect the fact that the productive reuse of software requires the software to be complemented with installation guides, metadata, product manuals, tutorials examples, documentation, and many other things. For describing these elements we will comply with the W3C ADMS Working Group [17] by referring to the term "asset." Assets are not limited to software but are considered abstract entities that reflect some "intellectual content independent of their physical embodiments" [17] or even more abstractly, an asset would be "a solution to a problem" [18]. Not every asset includes software: indeed, the Unity Asset Store offers a variety of solutions, either media assets, such as $3 \mathrm{D}$-objects, audio, textures, or particle systems, or software assets such as editor extensions, game AI, and physics engines.

The RAGE assets discussed in this paper are software assets. They may contain either a source code file or a compiled program file and may contain various other artefacts (cf. Figure 1).

The software artefact inside the asset is called "Asset Software Component." For being able to retrieve an asset from a wide variety of assets that are stored in an asset repository (the RAGE asset repository), it contains machinereadable metadata. In addition to keyword classifiers and asset descriptions the metadata include information about versions, asset dependencies, and programming language used among other things. The asset may include various additional artefacts that are not to be compiled (tutorials, manuals, licences, configuration tools, authoring tools, and other resources). While the constituents of the asset are stored separately in the RAGE repository, the asset can be packaged for distribution.

In the rest of this paper we will interchangeably use the term "Asset Software Component" and the term "asset" (as a shorthand notation) for indicating the software module that is to be linked or integrated with the game.

3.2. Basic Requirements. For being able to fulfil the ambition of the RAGE project to create an interoperable set of advanced game technology assets that can be easily used by serious game studios we have expressed the following basic requirements.

3.2.1. Interoperability between Assets. Since game developers may want to group multiple assets into more complex aggregates, data storage of assets to be used in their games and data exchange between assets should be well-defined.

3.2.2. Nomenclature. When creating a new asset, asset developers should not replicate functionalities of existing assets but instead should be able to exploit these. This requires a consistent approach to nomenclature of variable names, objects, and methods to be used across the whole set of assets, so that correct calls and references can be made.

3.2.3. Extendibility. The architecture should be robust over extending the set of assets with new assets. Developers may want to create new assets and add these to the existing set of assets. For allowing new assets to interact with existing ones, the interoperability conditions should be preserved.

3.2.4. Addressing Platform and Hardware Dependencies. Different hardware (e.g., game consoles) and operating systems may require different technical solutions. Browsers, actually the pairing of browsers and operating systems, display an even wider diversity with respect to browser brands, settings, and versions. This suggests that the programming for web browsers should be conservative as to avoid browser version issues as much as possible. Hence, direct access to the game user interface and/or operating system should be avoided.

3.2.5. Portability across Programming Languages. Using only well-established software patterns in RAGE would increase the portability as there will be valid existing code available from the start. Nevertheless, portability across different programming languages may be affected by the different nature and execution modes of the supported languages, for instance, interpreted languages such as JavaScript versus compiled languages as $\mathrm{C}++, \mathrm{C \#}$, and Java. Also multithreading versus single threading could hamper code portability.

3.2.6. Portability across Game Engines. The implementation of an asset in a supported programming language should be portable across different game engines and possibly even across hardware platforms and operating systems as much as possible. For this it is important to rely on the programming language's standard features and libraries to maximise the compatibility across game engines. Assets could thus delegate the implementation of required features to the actual game engine, for example, the actual storage of run-time data: as the game engine knows where and how data can be stored, assets could delegate the actual storage operation to the game engine and need not have their own stores.

3.2.7. Minimum Dependencies on External Software Frameworks. The usage of external software frameworks (such as jQuery or MooTools for JavaScript) should be avoided as much as possible because of their potential interference with the game engine code. Also namespace conflicts may readily occur when external frameworks were used.

3.2.8. Quality Assurance. For preserving the integrity of an ever-growing system of assets basic quality assurance requirements will be needed. With respect to coding RAGE assets require a consistent rather than mandatory coding style and expect documentation on class level or method level in order to accommodate debugging and maintenance. The inclusion of well-established software patterns and compliance with 


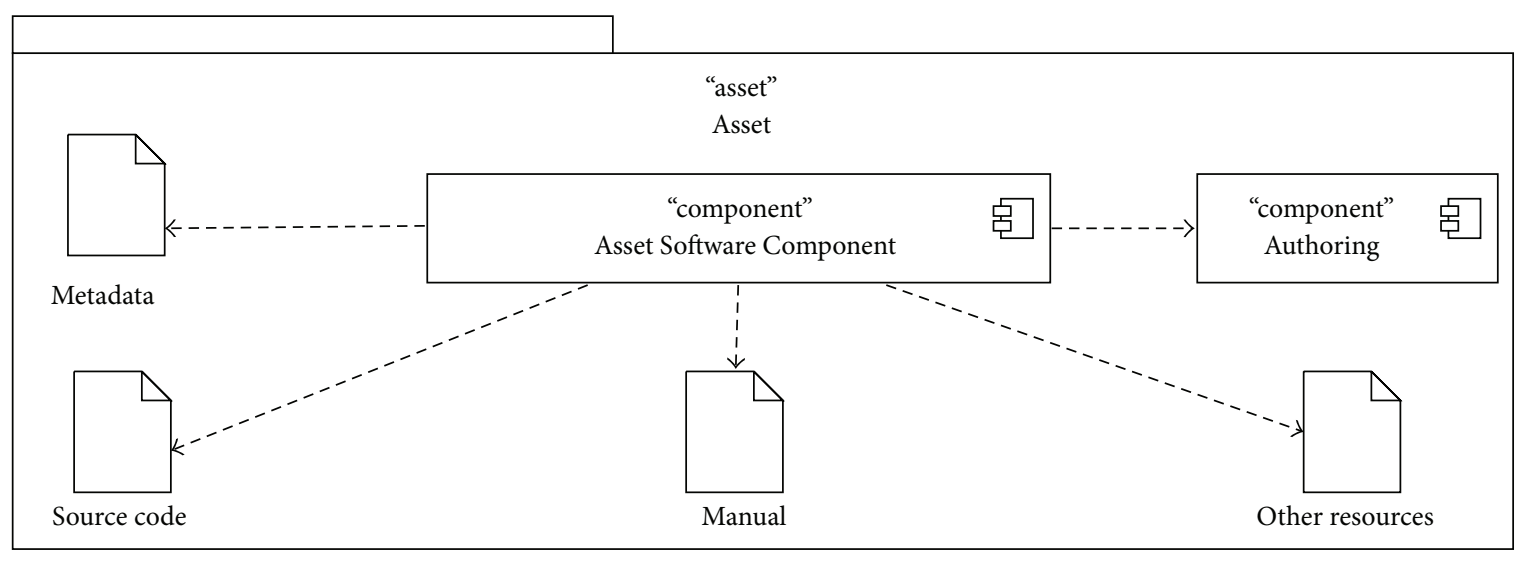

FIgURE 1: Exemplary layout of a RAGE asset.

(subsets of) existing standards in order to minimise overheads should have priority. Namespaces (or modules) should be used to prevent name conflicts between RAGE assets and game engine code.

3.2.9. Overall Simplicity. Software complexity should be avoided as to preserve practicability and maintainability. Preferably, assets should address only core functionality, which is in accordance with good practices of software development. Therefore, as a general starting point, the architecture and the integration points should be as simple as possible.

\section{Component-Based Design and Development}

Since RAGE assets are positioned as being used as reusable software components (plug-and-play), we adopt a component-based development approach (CBD) [19, 20]. In accordance with CBD, the RAGE asset architecture defines a component model for creating a reusable asset. The component model conforms to common norms of CBD, which are summarised as follows:

(i) A component is an independent and replaceable part of a system that fulfils a distinct function [20,21]. Following this norm, it is assumed that an asset provides a game developer with access to a concrete functionality that produces added value. For RAGE this added value will ideally (but not exclusively) be within the context of learning and teaching.

(ii) A component provides information hiding and serves as a black box to other components and technologies using it $[22,23]$. In accordance with this norm a game developer does not need to know how a particular functionality was implemented in the asset.

(iii) A component communicates strictly through a predefined set of interfaces that guard its implementation details $[20,21,24]$. Within the context of RAGE, it is assumed that a minimal intervention from a game developer is needed to integrate assets with a game engine.
Various existing CBD frameworks are available such as Enterprise JavaBeans [25] and CORBA [26]. However, we have chosen not to use these for several reasons. Although these frameworks simplify component integration and management, cross-platform support is still an issue. For example, Enterprise JavaBeans is limited to the Java platform. While CORBA is a language independent framework, mapping between different languages using an interface-definition language remains problematic. More critically, these frameworks are overly general and complex, which makes them unsuitable for addressing needs specific to the domain of serious game development. For example, game engines are de facto standard platforms for component control and reusability in game development, which creates a direct conflict with EJB or CORBA. For these and other reasons, existing CBD frameworks find very limited adoption in game development. RAGE will offer a less centralised approach by including a lightweight control component in the form of an AssetManager that is designed to complement game engines rather than take over their functionalities. The AssetManager is discussed in more detail later in this paper.

\section{RAGE Architectural Design}

5.1. Client-Side Assets versus Server-Side Assets. Assets can be positioned both client-side and server-side. Figure 2 sketches the general layout of an asset-supported game system.

On the client-side the player has access to a run-time game (game engine). The game may incorporate client-side assets that provide enhanced functionality. On the server-side a game server may be supported by server-side assets. Serverside assets may either be integrated with the game server or reside on remote servers.

Client-side assets should be preferred when frequent and direct interactions with the game engine on the local computer are required or when results should be immediately available. As a general guideline any processing that can be done client-side, for example, the processing of temporary, local data, should in fact be done clientside, as to reduce external communications as much as possible. However, when extensive processing is required, for 


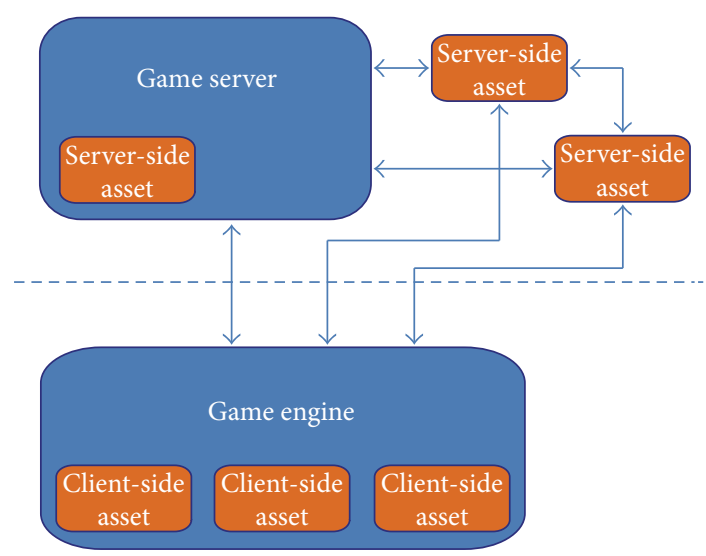

FIGURE 2: Distributed architecture of client-side assets and serverside assets supporting a game.

example, for speech recognition, a server-side asset would be appropriate in order to avoid poor game performance, for example, reduced frame rate or reduced game responsiveness. Obviously, assets that collect and process population data (learning analytics) and assets that require real time synchronisation across multiple remote players need to be server-side as well.

Communications between the game engine and the various servers are readily based on the http protocol, for example, REST and SOAP. On the client-side all assets are fully integrated with the game engine. This allows for direct interface methods, thus avoiding the impediments of SOA. The principal architectural challenges are on the client-side. The easy and seamless integration of multiple collaborating client-side assets as well as the portability of assets across multiple game engines, platforms, and programming languages requires a sound overall component architecture. This clientside architecture will be elaborated in the next sections.

5.2. Programming Languages. Given the wide variety of computer programming languages full portability of assets across programming languages will not be possible. It is inevitable to condense the spectrum of covered programming languages to those languages that are mostly used for creating games. This mainly holds for client-side code which is highly dictated by the game engine used, while server-side solutions are able to manage more diversity. For a start RAGE assets will be developed supporting two core code bases: C\# (for desktop and mobile games) and HTML5/JavaScript (for browser games), which are predominant products of compiled languages and interpreted languages, respectively. According to a survey among European game studios [27] the most popular programming language is C\# $(71 \%)$, followed by $\mathrm{C}++(67 \%)$, JavaScript $(48 \%)$, objective C $(33 \%)$, and Java $(33 \%)$, which is quite similar to the latest Redmonk programming languages rankings [28]. The growth of C\# has accelerated significantly since 2014, when Microsoft released its .NET core framework as open source, supporting any operating system and pushing the multiplatform nature of C\#. Major game engines or multiplatform tools (e.g.,
Unity/Xamarin) use C\# as their core language. $\mathrm{C}++$ is still being used a lot, but it is more complex than C\# or Java and often relies on platform-dependent constructs. With respect to browser-based games HTML5/JavaScript is considered a de facto standard. However, for overcoming the lack of strictness of JavaScript the RAGE project has adopted TypeScript (http://www.typescriptlang.org/) instead as the primary development language, which directly translates into JavaScript. TypeScript is used as a superclass of JavaScript that adds static typing, which can be used by integrated development environments and compilers to check for coding errors.

\subsection{The Asset Software Component's Internal Structure.} Figure 3 displays the UML class diagram of the Asset Software Component.

The classes, interfaces, and objects will be explained below. Code and detailed documentation of the asset implementation proofs are available on the GitHub repository at https://github.com/rageappliedgame. In particular we refer the C\# version (https://github.com/rageappliedgame/assetproof-of-concept-demo_CSharp), the TypeScript version (https://github.com/rageappliedgame/asset-proof-of-concept-demo_TypeScript), the JavaScript version (https:/github.com/rageappliedgame/asset-proof-of-concept-demo_JavaScript), the C++ version (https://github.com/rageappliedgame/asset-proof-of-concept-demo_CPlusPlus), and the Java version (https://github.com/rageappliedgame/asset-proof-ofconcept-demo_Java).

5.3.1. IAsset. The IAsset class, which is defined as an interface, provides the abstract definition of the Asset Software Component including the fields, properties, and methods required for its operations and communications.

5.3.2. BaseAsset. BaseAsset implements the set of basic functionalities following the definitions provided by IAsset. Moreover, BaseAsset exposes standardised interfaces that delegate the storage of component's default settings and runtime data used by the component to the game engine.

5.3.3. ISettings. In accordance with the abstract definition in the IAsset interface this interface ensures that every Asset Software Component has the basic infrastructure for managing a unique component ID, type, settings, version information, and so forth.

5.3.4. BaseSettings. This class realises the ISettings interface. It serves as a base class for the component's configuration settings.

5.3.5. IBridge. IBridge provides a standardised interface that allows the component to communicate with external technologies such as the game engine or a remote service.

5.3.6. ClientBridge. This bridge realises the IBridge interface. It mediates the direct communication from an Asset Software Component to the game engine (for calls from the game 


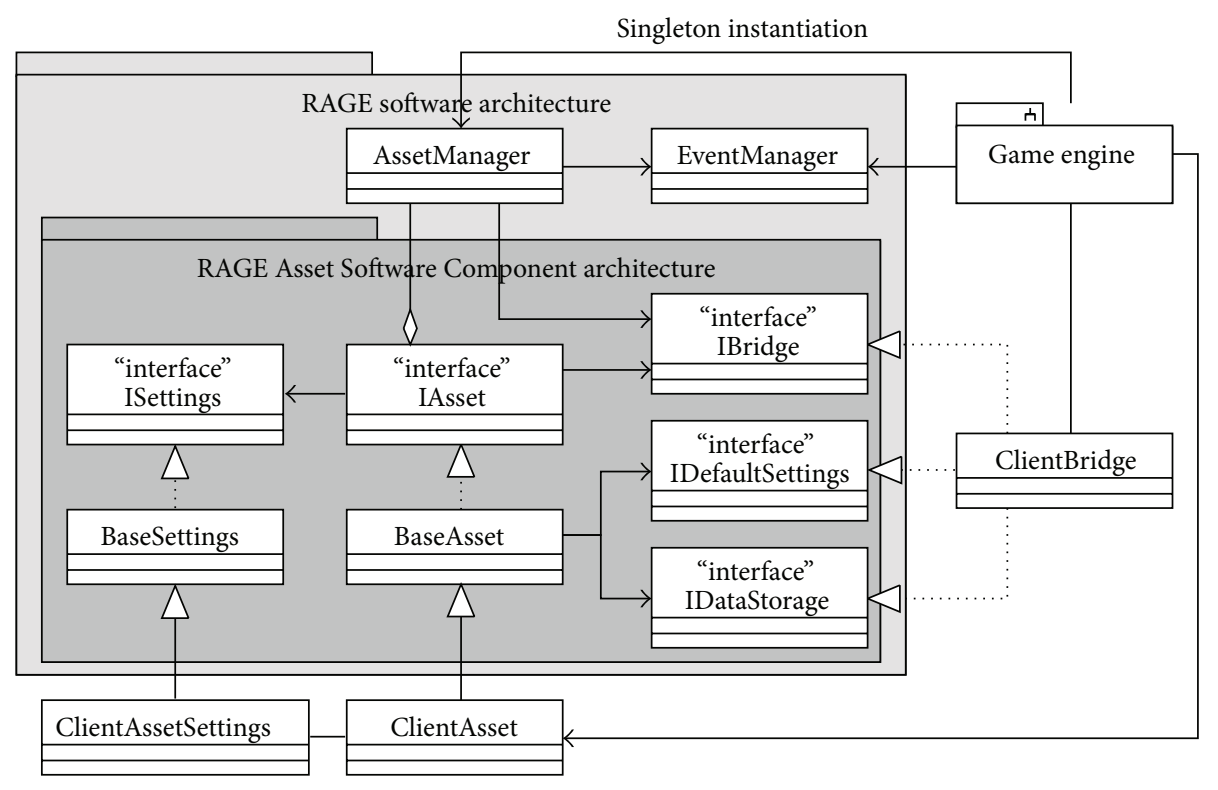

FIGURE 3: Class diagram reflecting the internal structure of an Asset Software Component.

engine to the component mediation is not required). In addition, ClientBridge may implement additional interfaces like IDataStorage via polymorphism. Thus, the same bridge object may provide the component with more specialised functionalities, such as allowing it to retrieve default settings from the game engine.

5.3.7. IDefaultSettings. The IDefaultSettings interface delegates the management of component's default settings to the game engine. It can be used when configuration parameters are stored in an external file rather than being hard-coded in a BaseSettings subclass.

5.3.8. IDataStorage. The IDataStorage interface delegates the management of run-time data used by the component to the game engine. It can be implemented to access a local file or to query a database.

5.3.9. AssetManager. The AssetManager takes over the management of multiple RAGE assets during both compile-time and run-time. The game engine instantiates all necessary assets and creates a singleton of the AssetManager that simplifies the tracking of all instantiated assets. The AssetManager covers the registration of the assets used by the game, it locates the registered assets on request, and it offers some common methods and events that may be used by the assets, for example, requesting the name and type of the game engine. The AssetManager is discussed in more detail in the next section.

5.3.10. EventManager. Alternatively, indirect (multicast) communication between various elements is also possible via the EventManager that is initialised by the AssetManager.
The RAGE asset architecture can thus be summarised as follows:

(i) The IAsset class defines the requirements for the RAGE asset as a reusable software component.

(ii) For game developers the implementation details of an Asset Software Component are hidden by a set of interfaces.

(iii) Likewise, the implementation details of an external technology are shielded for the Asset Software Component by one or more interfaces, for example, the storage of asset data in the game engine through IDataStorage, which is implemented on a bridge.

(iv) The architecture supports various means of communication, for example, between assets, via the bridge interfaces, or via the EventManager.

5.4. The AssetManager. As indicated above, the AssetManager is the coordinating agent for the management of multiple RAGE assets. Obviously, the AssetManager complies with the singleton pattern as only one coordinating agent is needed. The AssetManager covers the registration of all Asset Software Components that are used by the game engine. For achieving this it should expose methods to query this registration so that each Asset Software Component is able to locate other components and link to these. For avoiding duplicate code in each Asset Software Component the AssetManager could also be the provider of basic services that are relevant for all assets, such as the game engine's heartbeat, which indicates the game's proper operation, or user login/logout info for assets that need a user model. It centralises the code and requires only a single interaction point with the game engine. These data could be broadcast 
and transferred to Asset Software Components that have subscribed themselves for this event. Likewise, the AssetManager could also coordinate the link between the Asset Software Component and its data storage (which in fact requires reference to the game engine, because an Asset Software Component would not have storage capacity by itself).

5.5. Asset Communication. Asset Software Components need to communicate with the outside world, for instance, for receiving input from a user or game engine, for sending the results of their calculations to the game engine, for making web calls to query server-based services, or for linking up with other RAGE assets. For allowing an Asset Software Component (or its subcomponents) to communicate with the outside world, well-defined interfaces are needed. A set of standard software patterns and coding practices are used for accommodating these communications. These include the "Publish/Subscribe" pattern, the "bridge" pattern, Asset Method calls, and web services (cf. Table 1).

The various communication modes will be briefly explained below.

5.5.1. Asset Software Component's Methods. Each Asset Software Component may dynamically communicate with any other asset in the environment. This capability is enabled through registration by the AssetManager. An Asset Software Component can query the AssetManager for other Asset Software Components by referring to either a unique ID and/or a class name. Once the requested Asset Software Component is encountered, a direct communication can be established between two components without the need for further mediation by the AssetManager.

5.5.2. Bridges. For allowing an Asset Software Component to call a game engine method the bridge software pattern [29] is used, which is platform-dependent code exposing an interface. The game engine creates a bridge and registers it either at a specific asset or at the AssetManager. The BaseAsset then allows an Asset Software Component easy access to either one of these bridges to further communicate with the game engine. Overall, the bridge pattern is used to mediate a bidirectional communication between the Asset Software Component and the game engine while hiding game engine's implementation details. Additionally, polymorphism is used by allowing a bridge to implement multiple interfaces. The Asset Software Component may identify and select a suitable bridge and use its methods or properties to get the pursued game data.

5.5.3. Web Services. Communication through web services assumes an online connection to a remote service. Web services allow an Asset Software Component to call a service from the game engine by using a bridge interface. In principle, an Asset Software Component may not implement the communication interface itself and instead may rely on the adapters provided by the game engine [30]. Such approach would remove the asset's dependency on specific communication protocols used by remote services, thereby
TABLE 1: Communication modes of client-side assets.

\begin{tabular}{ll}
\hline $\begin{array}{l}\text { Client-side } \\
\text { requests }\end{array}$ & Communication modes \\
\hline Asset to asset & $\begin{array}{l}\text { These communications require a once-only } \\
\text { registration of the Asset Software Component at } \\
\text { the AssetManager: } \\
\text { (i) Publish/Subscribe } \\
\text { (ii) Asset Method call }\end{array}$ \\
\hline & $\begin{array}{l}\text { With bridge: } \\
\text { (i) Web services (e.g., outside world) }\end{array}$ \\
Asset to game & $\begin{array}{l}\text { (ii) Game engine functionality } \\
\text { engine }\end{array}$ \\
& $\begin{array}{l}\text { (iii) Hardware, operating system } \\
\text { Without bridge: }\end{array}$ \\
\hline Game engine & (i) Publish/Subscribe \\
to asset & (ii) Publish/Subscribe
\end{tabular}

allowing a greater versatility of the asset. Within the RAGE project automatic coupling with services will be supported by using the REST communication protocol [31]. When a service is unavailable, for example, when the game system is offline, the interface should be able to receive a call without processing it or acting on it. It is reminded here that serverside communications as indicated before in Figure 2 can all be implemented as web services.

5.5.4. Publish/Subscribe. Communications can also be arranged using the Publish/Subscribe pattern, which supports a 1-N type of communication (broadcasting). An example would be the game engine frequently broadcasting player performance data to multiple assets. The RAGE architecture allows for including Publish/Subscribe patterns where both Asset Software Components and the game engine can be either publishers or subscribers. The Publish/Subscribe pattern requires an EventManager, which is a centralised class that handles topics and events. The EventManager is initialised by the AssetManager during its singleton instantiation. Once initialised, either an asset or the game engine can use the EventManager to define new topics, (un)subscribe to existing topics, or broadcast new events. According to the Publish/Subscribe design pattern, subscribers do not have knowledge of publishers and vice versa. This allows an asset to ignore implementation details of a game engine or other assets. Additionally, this mode of communication is more suitable for asynchronous broadcasting to multiple receivers than the bridge-based communication, which realises bilateral communications only.

5.5.5. Composite Communications. The basic patterns explained above enable composite communication modes, which are composed of multiple stages. For instance, Asset Software Components may use the game engine as an intermediate step for their mutual communications, by using bridges, web services, or the Publish/Subscribe pattern. Also the AssetManager may act as a communication mediator. Once registered at the AssetManager, an Asset Software Component could use the AssetManager's set of commonly 
used methods and events in order to minimise the number of the game engine interaction points. In many cases it is more efficient to implement widely used functionality in the AssetManager than implementing it in every individual asset.

\section{Technical Validation}

For the technical validation of the RAGE architecture a basic software asset has been developed for all four of the selected programming languages. This basic Asset Software Component included all elementary operations and patterns, for example, registration, save, load, and log. The assets should meet the following test requirements:

(1) Once created, the Asset Software Components should induce the creation of a single AssetManager, which enables the components' self-registration.

(2) The AssetManager should be able to locate Asset Software Components and generate the associated versions and dependency reports.

(3) Asset Software Components should be able to directly connect through a method call.

(4) Asset Software Components should be able to call game engine functionality. The bridge code between the Asset Software Components and the game engine should provide some basic interfaces, such as simple file i/o and access to web services.

(5) The Publish/Subscribe pattern should allow Asset Software Components to both broadcast and subscribe to broadcasts, for example, transferring an object.

(6) The system should support multiple interactions, for example, a dialog system.

(7) The system should check for (default) settings and their serialisation to XML (for C\#) and should be able to include default settings at compile-time.

Tested implementation of the basic asset in all four programming languages can be found on GitHub (https://github .com/rageappliedgame). All implementation proved to meet the specified requirements. Yet, a number of languagedependent issues were encountered (and solved) that deserve further attention. In addition, the Unity game engine was used as an integration platform for the C\# asset version. A number of engine dependent issues were identified and solved as well.

\subsection{Issues in $\mathrm{C \#}$}

Characters. First, since Windows and OS X have different directory separator characters, forward slash (/) and backslash $(\backslash)$, respectively, portability fails. Problems can be avoided, however, by using the Environment class in C\# that dynamically returns the correct separator rather than using hard-coded separators. Second, the Mono version used in
Unity (v5.2) silently turns UTF-8 XML into UTF-16 during parsing, leading to problems during deserialisation of version info. This issue can be bypassed by omitting the parsing procedure and directly serialising the XML files.

Debugging. First, Mono's Debug.WriteLine method does not offer a syntax format such as String.Format. In order to obtain formatted diagnostic output messages during asset development, the String.Format method must be used explicitly. Second, debugging in Unity needs a different format of the debug symbol files generated during compilation with Visual Studio. Mono provides a conversion tool for this. Third, the pdb-to-mdb debug symbol converter of Unity (v5.2) cannot convert debug symbols created by Visual Studio 2015. A workaround is using Visual Studio 2013 or patch and recompile this utility. Finally, Mono and .Net display slight differences in the method names that are used for diagnostic logging. This problem can be solved easily by using the bridge for supplying the actual logging methods.

Compilation. In Unity the assemblies with embedded resources (i.e., RAGE Asset Software Components have their version data and localisation data embedded) cannot be compiled as upon compilation Unity automatically removes the embedded resources. The resources can be compiled with Visual Studio though, whereupon they can still be used in the Unity engine.

\subsection{Issues in TypeScript/JavaScript}

Characters. TypeScript and JavaScript rely on using a forward slash (/) directory separator on all platforms (Windows uses the backslash but allows using a forward slash). As TypeScript/JavaScript code will mainly be used for web-based games and will not try to access local files, this issue will be of little practical significance.

Interfaces. TypeScript implements interfaces but uses these only at compile-time for type checking. The resulting JavaScript is not capable of checking the existence of an interface as such. For allowing the asset to select the bridge to be used, the asset should instead check for the interface method that needs to be called.

Settings. During deserialisation of JSON data into asset settings JavaScript will only restore the data but will not recreate the methods present in the class. As a consequence computed properties will fail. A workaround is either to avoid using the methods and/or the computed values or to copy the restored data into a newly created settings instance.

\subsection{Issues in Java}

Characters. Because of the different directory separator characters in Windows and OS X, forward slash (/) and backslash $(\backslash)$, respectively, portability fails. Problems can be avoided by using the File.separator field, which dynamically returns the correct separator, instead of using hard-coded separators. 
Properties. Java does not support the properties concept of $\mathrm{C \#}$ and TypeScript but relies on naming conventions instead (get/set methods).

Default Values. Java has no standard implementation for default value attributes. As a consequence default values have to be applied in the constructor of the settings subclasses.

\subsection{Issues in $\mathrm{C}++$}

Characters. Also in C++ portability across Windows and OS $\mathrm{X}$ is hampered by the different directory separator characters. Problems can be avoided by creating conditional code for preprocessor directives that provide hard-coded separators tuned to the platform of compilation.

Properties. C++ does not support the concept of properties. Instead it relies on naming conventions (get/set methods).

Default Values. Like Java, C++ has no standard implementation for default value attributes, so default values have to be applied in the constructor of the settings subclasses.

Singleton. During testing the new $\mathrm{C}++2013$ singleton syntax led to crashes in Visual Studio, so it had to be replaced with a more traditional double-checked locking pattern.

Web Services. Although web service calls, for example, with REST or SOAP, are a well-established approach to clientserver communications and other remote communications, problems may arise because of slight semantic differences on different platforms. For instance, the popular JSON data format embedded in the web service protocols may suffer from this ambiguity, in particular with respect to the symbols of decimal separator, thousands separator, list separator, quote, data-time formats, null versus undefined, character encoding (e.g., UTF-8), prohibited characters in filenames, the line feed, and carriage return. XML-converted data are less sensitive to these issues.

\section{In Conclusion}

In this paper we have reported the design of the RAGE architecture, which is a reference architecture that supports the reuse of serious gaming technology components across different programming languages, game engines, and game platforms. An asset would offer a standardised interface that can be directly implemented by a game engine (via the bridge pattern) or it uses the asset's event manager for a Publish/Subscribe event. Proofs of concept in four principal code bases (C\#, Java, C++, and TypeScript/JavaScript) have validated the RAGE architecture. In addition, the C\# implementation of the test asset was successfully integrated in the Unity game engine, which demonstrates the practicability and validity of the RAGE asset architecture. The RAGE project will now start to develop up to 30 dedicated serious gaming assets and use these in customer-driven serious games projects. RAGE will make these assets available along with a large volume of high-quality knowledge resources on serious gaming through a self-sustainable delivery platform and social space. This platform aims to function as the single entry point for different stakeholders from the serious gaming communities, for example, game developers, researchers from multiple disciplines, online publishers, educational intermediaries, and end-users. RAGE thus aims to contribute to enhancing the internal cohesion of the serious games industry sector and to seizing the potential of serious games for teaching, learning, and various other domains.

\section{Competing Interests}

The authors declare that they have no competing interests.

\section{Acknowledgments}

This work has been partially funded by the EC H2020 project RAGE (Realising an Applied Gaming Ecosystem), http://www.rageproject.eu/, Grant Agreement no. 644187.

\section{References}

[1] T. M. Connolly, E. A. Boyle, E. MacArthur, T. Hainey, and J. M. Boyle, "A systematic literature review of empirical evidence on computer games and serious games," Computers \& Education, vol. 59, no. 2, pp. 661-686, 2012.

[2] J. M. Keller, "Development and use of the ARCS model of motivational design," Journal of Instructional Development, vol. 10, no. 3, pp. 2-10, 1987.

[3] J. M. Keller, "First principles of motivation to learn and e3learning," Distance Education, vol. 29, no. 2, pp. 175-185, 2008.

[4] R. M. Ryan and E. L. Deci, "Self-determination theory and the facilitation of intrinsic motivation, social development, and well-being," American Psychologist, vol. 55, no. 1, pp. 68-78, 2000.

[5] D. I. Cordova and M. R. Lepper, "Intrinsic motivation and the process of learning: beneficial effects of contextualization, personalization, and choice," Journal of Educational Psychology, vol. 88, no. 4, pp. 715-730, 1996.

[6] W. Westera, "Games are motivating, aren't they? Disputing the arguments for digital game-based learning," International Journal of Serious Games, vol. 2, no. 2, pp. 3-17, 2015.

[7] M. Polanyi, The Tacit Dimension, University of Chicago Press, Chicago, Ill, USA, 1966.

[8] W. Westera, R. J. Nadolski, H. G. K. Hummel, and I. G. J. H. Wopereis, "Serious games for higher education: a framework for reducing design complexity," Journal of Computer Assisted Learning, vol. 24, no. 5, pp. 420-432, 2008.

[9] S. Arnab, T. Lim, M. B. Carvalho et al., "Mapping learning and game mechanics for serious games analysis," British Journal of Educational Technology, vol. 46, no. 2, pp. 391-411, 2015.

[10] C. Linehan, B. Kirman, S. Lawson, and G. Chan, "Practical, appropriate, empirically-validated guidelines for designing educational games," in Proceedings of the ACM Annual SIGCHI Conference on Human Factors in Computing Systems (CHI '11), pp. 1979-1988, ACM, Vancouver, Canada, May 2011.

[11] J. Stewart, L. Bleumers, J. Van Looy et al., The Potential of Digital Games for Empowerment and Social Inclusion of Groups at Risk of Social and Economic Exclusion: Evidence and Opportunity for 
Policy, Joint Research Centre, European Commission, Brussels, Belgium, 2013.

[12] R. García Sánchez, J. Baalsrud Hauge, G. Fiucci et al., "Business Modelling and Implementation Report 2," GALA Network of Excellence for Serious Games, 2013, http://www.galanoe.eu.

[13] E. Folmer, "Component based game development-a solution to escalating costs and expanding deadlines?" in ComponentBased Software Engineering, pp. 66-73, Springer, Berlin, Germany, 2007.

[14] A. W. B. Furtado, A. L. M. Santos, G. L. Ramalho, and E. S. De Almeida, "Improving digital game development with software product lines," IEEE Software, vol. 28, no. 5, pp. 30-37, 2011.

[15] M. B. Carvalho, F. Bellotti, J. Hu et al., "Towards a serviceoriented architecture framework for educational serious games," in Proceedings of the 15th IEEE International Conference on Advanced Learning Technologies (ICALT '15), pp. 147-151, IEEE, Hualien, Taiwan, July 2015.

[16] M. B. Carvalho, F. Bellotti, R. Berta et al., "A case study on service-oriented architecture for serious games," Entertainment Computing, vol. 6, pp. 1-10, 2015.

[17] M. Dekkers, Asset Description Metadata Schema (ADMS), W3C Working Group, 2013, http://www.w3.org/TR/vocab-adms/.

[18] Object Management Group, Reusable Asset Specification, Version 2.2, 2005, http://www.omg.org/spec/RAS/2.2/.

[19] F. Bachmann, L. Bass, C. Buhman et al., Volume II: Technical Concepts of Component-based Software Engineering, Carnegie Mellon University, Software Engineering Institute, Pittsburgh, Pa, USA, 2000.

[20] S. Mahmood, R. Lai, and Y. S. Kim, "Survey of componentbased software development," IET Software, vol. 1, no. 2, pp. 5766, 2007.

[21] X. Cai, M. R. Lyu, K. F. Wong, and R. Ko, “Component-based software engineering: technologies, development frameworks, and quality assurance schemes," in Proceedings of the 7th AsiaPacific Software Engineering Conference (APSEC '00), pp. 372379, IEEE, Singapore, 2000.

[22] K. K. Lau and F. M. Taweel, "Data encapsulation in software components," in Component-Based Software Engineering, pp. 116, Springer, Berlin, Germany, 2007.

[23] T. Wijayasiriwardhane, R. Lai, and K. C. Kang, "Effort estimation of component-based software development-a survey," IET Software, vol. 5, no. 2, pp. 216-228, 2011.

[24] H. Koziolek, "Performance evaluation of component-based software systems: a survey," Performance Evaluation, vol. 67, no. 8, pp. 634-658, 2010.

[25] E. Roman, R. P. Sriganesh, and G. Brose, Mastering Enterprise JavaBeans, John Wiley \& Sons, 2005.

[26] S. Vinoski, "CORBA: integrating diverse applications within distributed heterogeneous environments," IEEE Communications Magazine, vol. 35, no. 2, pp. 46-55, 1997.

[27] G. L. Saveski, W. Westera, L. Yuan et al., "What serious game studios want from ICT research: identifying developers' needs," in Proceedings of the Games and Learning Alliance conference (GALA '15), Serious Games Society, Rome, Italy, December 2015.

[28] Redmonk programming languages rankings, 2015, http://redmonk.com/sogrady/2015/01/14/language-rankings-1-15/.

[29] E. Gamma, R. Helm, R. Johnson, and J. Vlissides, Design Patterns: Elements of Reusable Object-Oriented Software, Pearson Education, 1994.
[30] B. Benatallah, F. Casati, D. Grigori, H. R. M. Nezhad, and F. Toumani, "Developing adapters for web services integration," in Advanced Information Systems Engineering, O. Pastor and J. Falcão e Cunha, Eds., vol. 3520 of Lecture Notes in Computer Science, pp. 415-429, Springer, Berlin, Germany, 2005.

[31] A. P. Sheth, K. Gomadam, and J. Lathem, "SA-REST: semantically interoperable and easier-to-use services and mashups," IEEE Internet Computing, vol. 11, no. 6, pp. 91-94, 2007. 


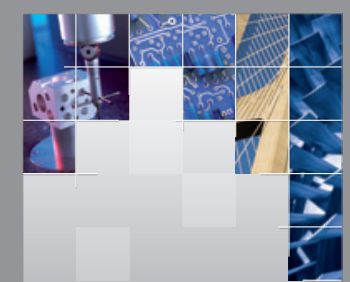

\section{Enfincering}
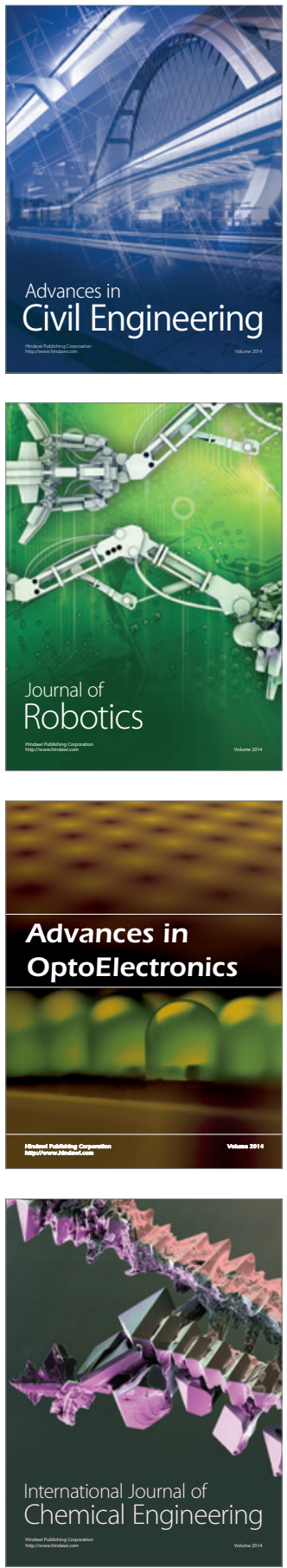

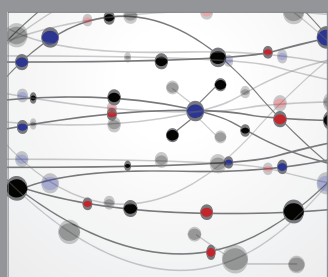

The Scientific World Journal

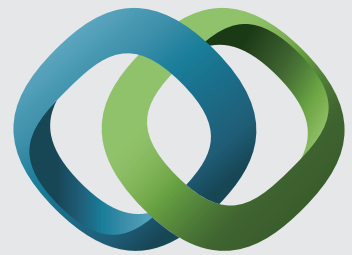

\section{Hindawi}

Submit your manuscripts at

http://www.hindawi.com
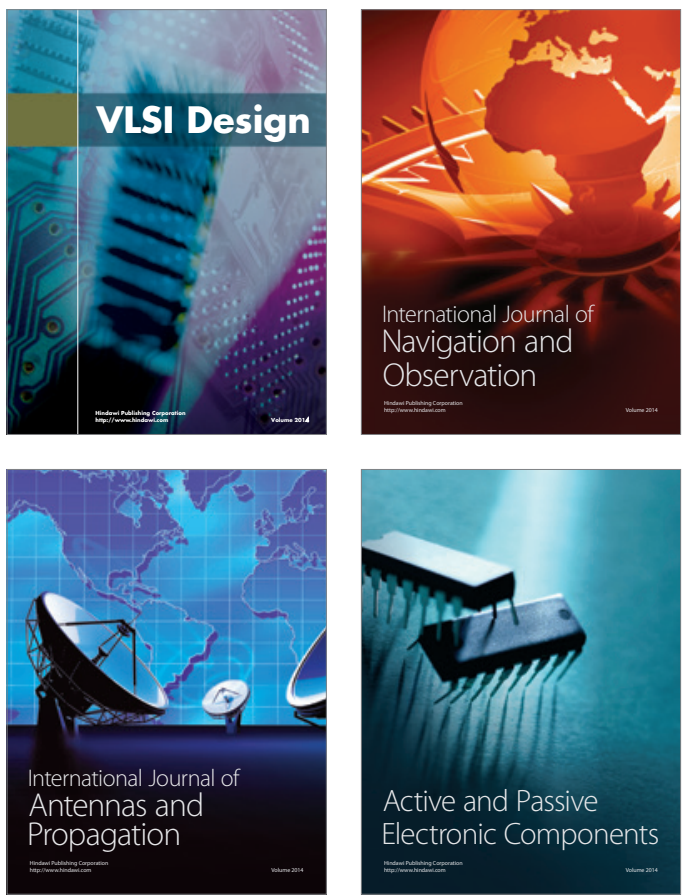
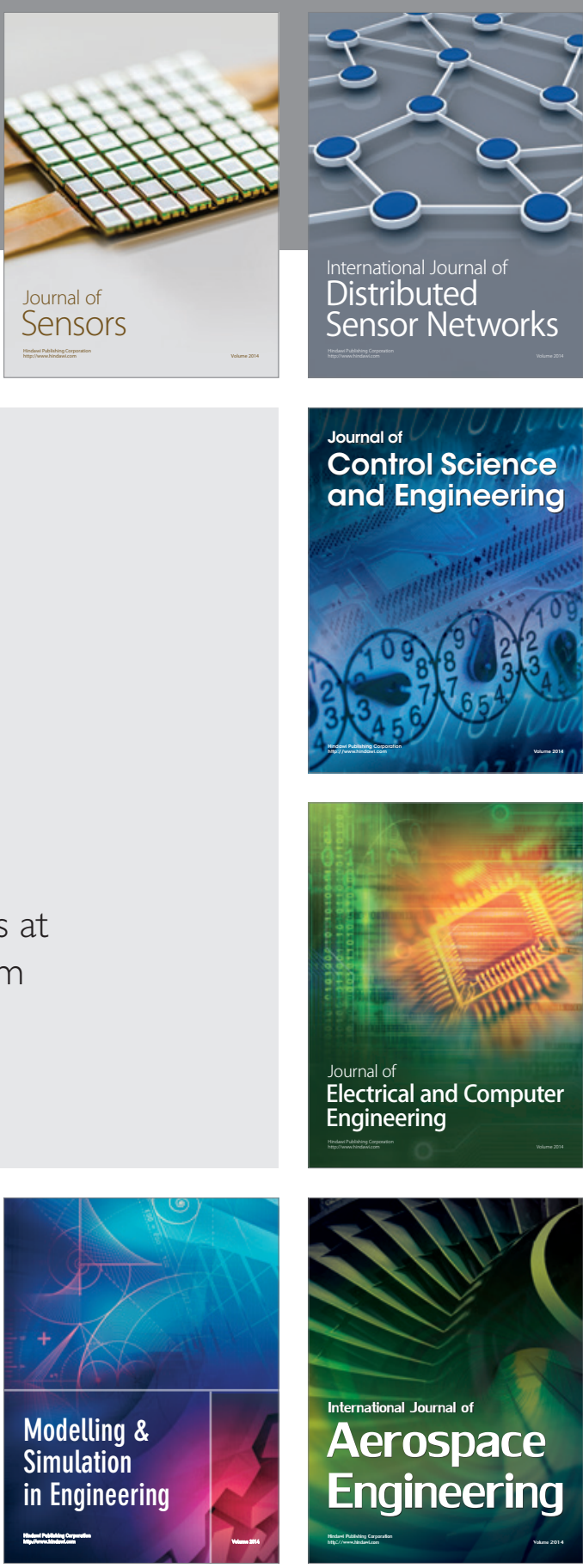

International Journal of

Distributed

Sensor Networks

Journal of

Control Science

and Engineering
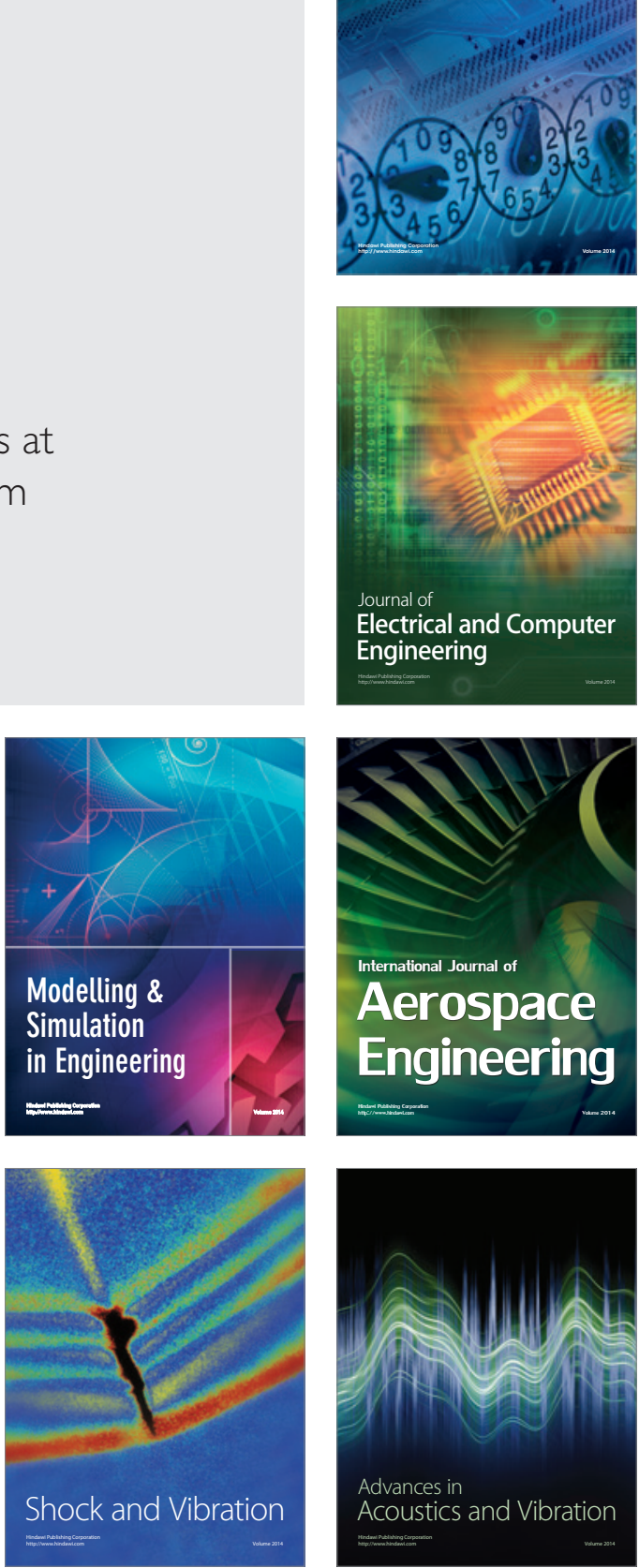